\title{
Glionitrin A, a New Diketopiperazine Disulfide, Activates ATM-ATR- Chk1/2 via 53BP1 Phosphorylation in DU145 Cells and Shows Antitumor Effect in Xenograft Model
}

\author{
Young-Joo Kim, Hyun Bong Park, Ji-Hye Yoo, Hak Cheol Kwon, Joonki Kim, and \\ Hyun Ok Yang* \\ Natural Medicine Center, Korea Institute of Science and Technology; 679 Saimdang-ro, Gangneung, Gangwon-do \\ 210-340, Republic of Korea. \\ Received September 12, 2013; accepted December 8, 2013
}

In a recent study, we isolated the diketopiperazine disulfide glionitrin A from the co-culture broth of a mine drainage-derived fungus (Aspergillus fumigatus KMC901) and bacterium (Sphingomonas KMK001). Here, we investigated the antitumor activity of glionitrin A and its underlying molecular mechanisms in human prostate cancer DU145 cells. Glionitrin A showed significant cytotoxicity, promoting cell cycle arrest and apoptosis. Glionitrin A-treated cells exhibited elevated levels of phospho-histone 2AX (Ser139), a marker of DNA damage, and accumulated in both $S$ phase and G2/M phase due to the activation of checkpoints associated with the ataxia-telangiectasia-mutated and ataxia-telangiectasia-mutated-Rad3-related Chk1/2 pathway downstream of p53-binding protein 1 phosphorylation at Ser1778. In addition, glionitrin A induced apoptosis through both caspase-dependent and -independent pathways. Glionitrin A activated caspase-8, -9 and $\mathbf{- 3}$ and also released endonuclease $G$ from the mitochondria to the nucleus in a dose-dependent manner. Our in vivo study performed in nude mice bearing xenografts of DU145 cells showed that glionitrin A dramatically reduced the tumor volume by an average of $38.2 \%(5 \mathrm{mg} / \mathrm{kg}$, per os (p.o.)) and $71.3 \%(10 \mathrm{mg} / \mathrm{kg}$, p.o.) at $27 \mathrm{~d}$ after the beginning of treatment. Taken together, these findings provide a detailed description of the mechanism underlying the biological activity of the new natural product glionitrin $A$, which has the potential to be developed as an anti-prostate cancer agent.

Key words glionitrin A; DNA damage; 53BP1; prostate cancer; xenograft

Glionitrin A is a novel diketopiperazine metabolite that was isolated from a co-culture of the bacterial strain Sphingomonas KMK-001 and the fungal strain Aspergillus fumigatus KMC-901, which were isolated from acidic coal mine drainage. ${ }^{1)}$ Glionitrin A was not detected in mono-culture broths of either microbe under the same conditions. The structure of glionitrin A is closely related to that of gliotoxin, which is the major compound produced by Aspergillus fumigatus KMC-901, except that it contains a nitro functional group on its aromatic ring and its disulfide chiral centers have the opposite absolute configurations of those of gliotoxin, as determined by circular dichroism and X-ray crystallography. Gliotoxin is a representative fungal mycotoxin from a class of epidithiodiketopiperazines (ETPs). ${ }^{2)}$ It is produced by various fungal strains, such as Aspergillus fumigates, Gliocladium fimbriatum and Penicillium spp., and it exerts a variety of biological activities, including antibiotic, antiangiogenic, immunosuppressive and apoptosis-inducing effects. ${ }^{3)}$ Due to its strong cytotoxic activity, gliotoxin is considered as a potential antitumor agent. In addition, glionitrin A displays potent antimicrobial activity against several pathogenic bacteria, including methicillin-resistant Staphylococcus aureus, and shows powerful cytotoxicity against various human cancer cell lines. ${ }^{1)}$ However, its mechanisms of action are not yet understood.

Apoptosis is a critical aspect of the cellular response to anti-cancer compounds. ${ }^{4)}$ Unicellular organisms respond to DNA lesions by activating cell cycle checkpoints and repair pathways, while multicellular organisms respond to DNA damage by eliminating the damaged cells by activating apo-

The authors declare no conflict of interest ptosis. $^{5,6)}$ Genome integrity and stability is continuously threatened by DNA damage, which may be induced by numerous environmental and endogenous causes. The failure to repair DNA damage can cause cancer by introducing mutations and genomic instability. ${ }^{7}$ The DNA damage response consists of multiple networks of signal transduction pathways that are initiated by the sensing of DNA damage, followed by signal transmission to effectors through a phosphorylation cascade that is executed by two phosphoinositide 3-kinase related kinase (PIKK) family members, ataxia-telangiectasia mutated (ATM) and ATM- and Rad3-related (ATR). ${ }^{8}$ ) The checkpoint functions of ATM and ATR are mediated in part by checkpoint effector kinases, such as checkpoint kinase 1 (Chk1) and Chk2. Activated Chk1 and Chk2 phosphorylate cell division cycle 25 (cdc25) family phosphatases, leading to their degradation and ultimately cell cycle arrest. ${ }^{9,10)}$ In addition, ATR and ATM amplify the damage signal by phosphorylating numerous substrates, including histone 2AX (H2A.X), Nijmegen breakage syndrome 1 (NBS1) and structural maintenance of chromosomes 1 (SMC1), recruiting chromatin remodeling complexes to the sites of double-strand breaks (DSBs) in the DNA. ${ }^{9,11,12)}$ Meanwhile, ATM also induces p53 phosphorylation, which activates pro-apoptotic genes, such as Bcl-2-associated X protein (Bax), p53 up-regulated modulator of apoptosis (PUMA) and the Fas receptor. ${ }^{13)}$ Therefore, the activation of the ATM/ATR-p53 cascade is an important event in DNA damage-induced apoptosis. DNA damage also activates p53-independent apoptosis through backup systems. ${ }^{14)}$ ATM/ATR-Chk1/2 signaling upon DNA damage activates E2F transcription factor 1 (E2F1), leading to an increase in the level of $\mathrm{p73}{ }^{15)}$ The upregulation of $\mathrm{p} 73$ activates NOXA, PUMA and Bax, resulting in apoptosis. ${ }^{16)}$ 
In the present study, we demonstrated the cytotoxicity of glionitrin A in DU145 cells and characterized the DNA damage-related cellular and biochemical mechanisms underlying this effect.

\section{MATERIALS AND METHODS}

Chemicals and Antibodies The isolated compound (Fig. 1A) was confirmed as glionitrin A by comparing the ${ }^{1} \mathrm{H}$ - and ${ }^{13} \mathrm{C}-\mathrm{NMR}$, optical rotation and electrospray ionization mass spectrometry (ESI-MS) data from the compound with previously published values. ${ }^{1)}$ The purity of this compound was greater than $99 \%$, based on HPLC analysis. A stock solution of glionitrin A was dissolved in 100\% dimethyl sulfoxide (DMSO) and stored at $-20^{\circ} \mathrm{C}$. When required, the stock solution was diluted with cell culture media to the appropriate concentration. The final concentration of DMSO in the culture media was adjusted to $0.5 \%(\mathrm{v} / \mathrm{v})$. Caffeine, which was used as an ATM/ATR inhibitor, and paclitaxel were obtained from Sigma-Aldrich (St. Louis, MO, U.S.A.). Z-VAD-fmk, a general caspase inhibitor, was purchased from R\&D Systems (Min-

A<smiles>CN1C(=O)C23Cc4ccc([N+](=O)[O-])cc4N2C(=O)C1C3CO</smiles>

Glionitrin A

C



$\mathbf{E}$



neapolis, MN, U.S.A.).

Primary antibodies against phospho-H2A.X (Ser139), phospho-53BP1 (S11778), 53BP1, phospho-ATM (Ser1981), phospho-ATR (Ser428), ATR, phospho-Chk1 (Ser317), phospho-Chk1 (Ser345), Chk1, phospho-Chk2 (Thr68), phosphoChk2 (Thr387), Chk2, cdc25A, phosphor-cdc2 (Tyr15), cdc2, cleaved caspase-8, Bid, Bax, cleaved caspase-9, cleaved caspase-3, poly(ADP-ribose) polymerase (PARP), apoptosisinducing factor (AIF), endonuclease $G$ (EndoG), histone deacetylase 1 (HDAC1), cytochrome $c$ oxidase subunit IV (COX IV) and glyceraldehyde-3-phosphate dehydrogenase (GAPDH) and peroxidase-conjugated secondary antibodies were purchased from Cell Signaling Technology (Danvers, MA, U.S.A.). The antibody against ATM was obtained from Rockland Immunochemicals (Gilbertsville, PA, U.S.A.). The antibody against phospho-BID was obtained from Novus Biologicals (Littleton, CO, U.S.A.). The Alexa Fluor 488-labeled secondary antibody was purchased from Molecular Probes (Eugene, OR, U.S.A.).

Cell Culture The human prostate cancer cell line DU145 was purchased from the American Type Culture Collection

\section{B}

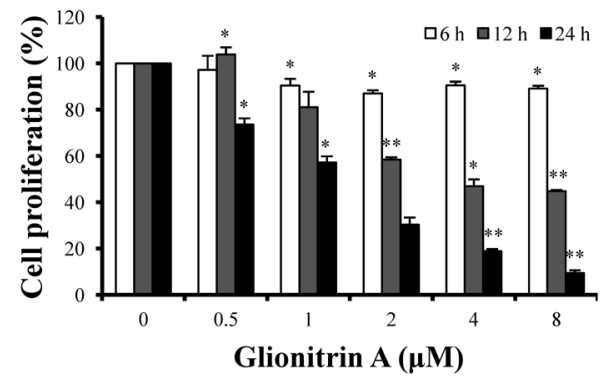

D
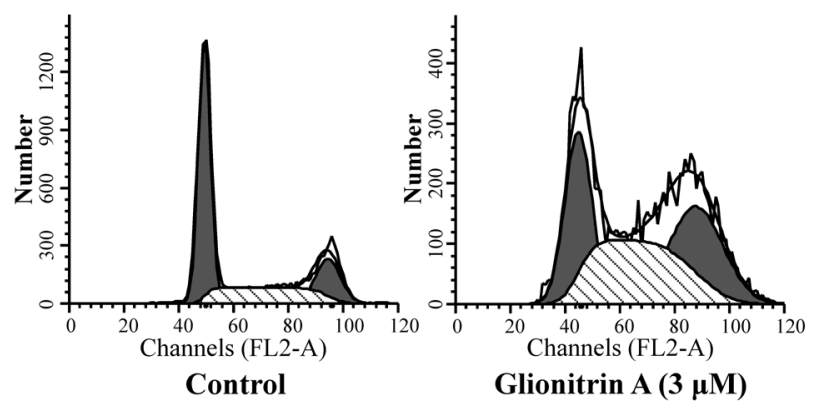

F
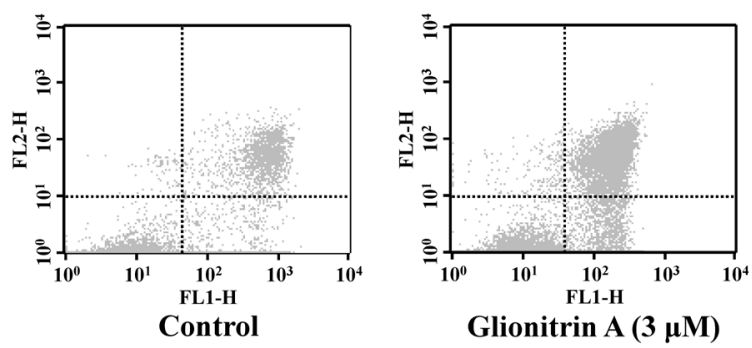

Fig. 1. Glionitrin A Causes Cytotoxicity, Cell Cycle Arrest and Apoptosis in DU145 Cells

(A) Chemical structure of glionitrin A. (B) Cells were treated with the indicated concentrations of glionitrin A for 6,12 and $24 \mathrm{~h}$, and cell proliferation was examined by CCK-8 assay. (C) Cells were treated with varying concentrations of glionitrin A for 12 and $24 \mathrm{~h}$, and FACS analysis/PI staining was then performed to assess cell cycle distribution. (D) Representative FACS diagrams of PI staining at $24 \mathrm{~h}$. (E) Cells were treated with $3 \mu \mathrm{M}$ glionitrin A for 6,12 and $24 \mathrm{~h}$, and FACS analysis/AV-PI doublestaining was then performed to assess apoptotic cell death. (F) Representative FACS diagrams of AV/PI double-staining at $24 \mathrm{~h}$. Each value represents the mean \pm S.E.M. of three independent experiments. ${ }^{*} p<0.05$ and $* * p<0.01$ compared with the control. 
(ATCC, Manassas, VA, U.S.A.). The cells were cultured in MEM (Gibco BRL, Carlsbad, MD, U.S.A.) supplemented with $10 \%$ fetal bovine serum (Gibco BRL), $100 \mathrm{U} / \mathrm{mL}$ penicillin and $100 \mu \mathrm{g} / \mathrm{mL}$ streptomycin and incubated at $37^{\circ} \mathrm{C}$ in a humidified atmosphere with $5 \% \mathrm{CO}_{2}$.

Cell Proliferation Assay Cell Counting Kit-8 (Dojindo Laboratories, Kumamoto, Japan) was used to evaluate cell proliferation. Control cells were exposed to culture media containing $0.5 \%$ (v/v) DMSO. Paclitaxel was used as a positive control (data not shown).

Flow Cytometry Analysis To detect changes in cell cycle distribution, the treated cells were collected, washed with cold phosphate buffered saline (PBS) and fixed in 70\% ethanol at $4^{\circ} \mathrm{C}$ for $30 \mathrm{~min}$. The cells were then washed twice with PBS and resuspended in $500 \mu \mathrm{L}$ of a propidium iodide (PI) staining solution containing $40 \mu \mathrm{g} / \mathrm{mL}$ PI and $20 \mu \mathrm{g} / \mathrm{mL}$ RNase A in PBS. Then, the cells were incubated at room temperature for $30 \mathrm{~min}$ in the dark and analyzed with a FACSCalibur flow cytometer (Becton-Dickinson, San Jose, CA, U.S.A.) and the ModFit LT V2.0 computer program.

Apoptotic cell death was assessed by annexin-V/PI double staining using an Annexin-V-FLUOS Staining Kit (Roche, Penzberg, Germany) according to the manufacturer's recommendations. At least 20000 events were evaluated in each experiment.

Single-Cell Gel Electrophoresis Assay (Comet Assay) To detect double-stranded DNA breaks (DSBs), the treated cells were embedded in agarose and electrophoresed according to the manufacturer's instructions (Trevigen, Gaithersburg, MD, U.S.A.). Control cells were exposed to culture media containing $0.5 \%(\mathrm{v} / \mathrm{v})$ DMSO.

Preparation of Whole-Cell Extracts Whole-cell extracts were prepared according to the manufacturer's instructions in RIPA buffer (Cell Signaling) supplemented with $1 \times$ protease inhibitor cocktail and $1 \mathrm{~mm}$ phenylmethylsulfonyl fluoride (PMSF).

Preparation of Cytosolic and Nuclear Extracts The treated cells were rinsed twice with cold PBS and collected. The cell pellets were resuspended in a hypotonic buffer (10 mM 4-(2-hydroxyethyl)-1-piperazineethanesulfonic acid (HEPES) [pH 7.9], $10 \mathrm{~mm} \mathrm{KCl,} 0.1 \mathrm{~mm}$ ethylenediaminetetraacetic acid (EDTA), $1 \mathrm{~mm}$ dithiothreitol (DTT), $1 \times$ protease inhibitor cocktail, $1 \mathrm{~mm}$ PMSF and $\left.1 \mathrm{~mm} \mathrm{Na}_{3} \mathrm{VO}_{4}\right)$ for $15 \mathrm{~min}$ on ice, after which $10 \%$ Nonidet P-40 (USB, OH, U.S.A.) was added. The mixture was vortexed and then centrifuged at $13200 \mathrm{rpm}$ for $30 \mathrm{~s}$ at $4^{\circ} \mathrm{C}$. The supernatant, which contained the cytosolic proteins, was collected and stored at $-80^{\circ} \mathrm{C}$ until further use. The nuclear pellets were rinsed twice with cold PBS and resuspended in a hypertonic buffer $(20 \mathrm{~mm}$ HEPES [pH 7.9], $0.4 \mathrm{M} \mathrm{NaCl}, 0.1 \mathrm{~mm}$ EDTA, $1 \mathrm{~mm}$ DTT, $1 \times$ protease inhibitor cocktail, $1 \mathrm{~mm}$ PMSF and $1 \mathrm{~mm} \mathrm{Na} \mathrm{NO}_{4}$ ) by rocking at $4^{\circ} \mathrm{C}$ for $15 \mathrm{~min}$. The resuspended nuclear fraction was then centrifuged at $13200 \mathrm{rpm}$ for $5 \mathrm{~min}$ at $4^{\circ} \mathrm{C}$. The supernatant, which contained the nuclear proteins, was collected and stored at $-80^{\circ} \mathrm{C}$ until further use.

Immunoblot Analysis Following the desired treatments, cell lysates (whole-cell extracts: $30 \mu \mathrm{g} /$ lane, nuclear extracts: $10 \mu \mathrm{g} /$ lane, cytosolic extracts: $30 \mu \mathrm{g} /$ lane) were prepared and then separated by electrophoresis on a NuPAGE 4-12\% BisTris gel (Invitrogen, Carlsbad, CA, U.S.A.), blotted onto a polyvinylidene difluoride (PVDF) transfer membrane and ana- lyzed with epitope-specific primary and secondary antibodies. The bound antibodies were visualized using ECL Advance Western Blotting Detection Reagents (GE Healthcare, Buckinghamshire, U.K.) and a LAS-4000 system (FUJIFILM, Tokyo, Japan).

Caspase Activity Assay The activities of caspase-3, -8 and -9 were assessed using a Fluorometric Assay Kit (BioVision, Milpitas, CA, U.S.A.) according to the manufacturer's protocol.

Confocal Microscopy DU145 cells were seeded onto sterile coverslips in 12-well plates. On the following day, the cells were pretreated with or without $20 \mu \mathrm{M}$ Z-VAD-fmk for $2 \mathrm{~h}$ and then exposed to $3 \mu \mathrm{M}$ glionitrin A for $24 \mathrm{~h}$. For mitochondrial labeling, the cells were incubated in PBS containing $100 \mathrm{~nm}$ MitoTracker Red CMXRos (Molecular Probes) for $20 \mathrm{~min}$ under normal growth conditions. The cells were then fixed with $4 \%$ paraformaldehyde for $20 \mathrm{~min}$, permeabilized with $0.2 \%$ Triton X-100 for $30 \mathrm{~min}$ and blocked with $2 \%$ BSA in PBS for $1 \mathrm{~h}$. The fixed cells were sequentially incubated with an anti-EndoG primary antibody at room temperature for $1 \mathrm{~h}$, an Alexa Fluor 488-conjugated secondary antibody at room temperature for $1 \mathrm{~h}$ in the dark and $1 \mu \mathrm{g} / \mathrm{mL} \mathrm{4,'6'-diamidino-}$ 2-phenylindole (DAPI) at room temperature for $20 \mathrm{~min}$ in the dark. The coverslips were placed on slides that had been precoated with a drop of ProLong Gold Antifade Reagent (Invitrogen) and sealed using nail lacquer. Images were obtained using a Leica TCS SP5 confocal microscope (Leica, Germany). Control cells were treated with culture media containing $0.5 \%(\mathrm{v} / \mathrm{v})$ DMSO.

In Vivo Tumor Xenograft Study Five weeks-old male BALB/c-nu mice (17-22g) were obtained from SLC (Japan). After two weeks of adaptation period, each mouse received a subcutaneous injection of $1 \times 10^{7}$ DU145 cells into the right flank. When tumors developed to a size of approximately $80 \mathrm{~mm}^{3}$, the mice were randomly allocated into four groups (control, cisplatin $4 \mathrm{mg} / \mathrm{kg}$, glionitrin A 5 and $10 \mathrm{mg} / \mathrm{kg}$ ). Each group contained seven animals. Glionitrin A was prepared in deionized water (DIW) with $2.5 \%$ Tween 80 and $2.5 \%$ ethanol and orally administered to the mice at a dose of 5 or $10 \mathrm{mg} / \mathrm{kg}$ twice per week for two weeks. Cisplatin was prepared in sterile saline and administered intravenously at a dose of $4 \mathrm{mg} / \mathrm{kg}$ once per week for three weeks. The animals in the control group received DIW containing 2.5\% Tween 80 and $2.5 \%$ ethanol with sterile saline. The tumor volumes and body weights were monitored twice per week for the entire duration of the experiment. The tumor volumes were calculated using the formula $\left[(W)^{2} \times L\right] / 2$, where $W$ and $L$ are the width (short diameter) and length (long diameter) of the tumor, respectively. At the end of the experiment, all of the animals were sacrificed for tumor collection. All animal studies were performed in accordance with the guidelines of the Institutional Animal Care and Use Committee of the Korea Institute of Science and Technology.

Statistical Analysis The results are expressed as the means \pm S.E.M. of at least three independent determinations for each experiment. Statistical significance was analyzed using Student's $t$-tests. The $p$ value of $<0.05$ was considered significant. 


\section{RESULTS}

The Effect of Glionitrin A on Cell Proliferation, Cell Cycle Progression and Apoptotic Cell Death In previous studies assessing the efficacy of glionitrin A in various human cancer cell lines, the drug showed the strongest inhibition of cell proliferation in human prostate cancer cells. ${ }^{1)}$ Based on this pilot observation, we focused on DU145 cells to examine the antitumor effect of glionitrin A in this study.

The antiproliferative activity of glionitrin A in DU145 cells was evaluated over a 24-h time course. As shown in Fig. 1B, glionitrin A was significantly cytotoxic, with $\mathrm{IC}_{50}$ values of $3.1 \pm 0.36$ and $1.5 \pm 0.28 \mu \mathrm{M}$ at 12 and $24 \mathrm{~h}$, respectively, while its effect was relatively weak at $6 \mathrm{~h}$. We next investigated whether the cell proliferation inhibition was accompanied by changes in cell cycle progression and/or apoptosis induction. The effect of glionitrin A on the cell cycle distribution at 12 and $24 \mathrm{~h}$ is shown in Figs. 1C and 1D. The population of cells in $\mathrm{S}$ phase was increased by treatment with glionitrin $\mathrm{A}$ at $0.75,1.5$ and $3 \mu \mathrm{M}$ in a dose-dependent manner at both 12 and $24 \mathrm{~h}$ of treatment $(30.4 \pm 2.08,32.9 \pm 3.12$ and $33.1 \pm$ $5.20 \%$ versus $21.3 \pm 3.07 \%$ at $12 \mathrm{~h} ; 26.7 \pm 5.08,36.2 \pm 6.12$ and $45.7 \pm 7.20 \%$ versus $25.8 \pm 0.07 \%$ at $24 \mathrm{~h}$ ). The population of cells in G2/M phase was also elevated under the same conditions, although the greatest increase was observed at the lowest dose of glionitrin A $(22.0 \pm 1.39,20.0 \pm 3.54$ and $18.7 \pm$ $1.80 \%$ versus $17.5 \pm 2.19 \%$ at $12 \mathrm{~h} ; 36.8 \pm 1.39,30.2 \pm 6.54$ and $27.9 \pm 0.80 \%$ versus $18.7 \pm 2.19 \%$ at $24 \mathrm{~h}$ ). We next assessed the effects of glionitrin A on apoptotic cell death. Exposure to $3 \mu \mathrm{M}$ glionitrin A resulted in significant apoptotic death at $24 \mathrm{~h}$ (Figs. $1 \mathrm{E}$ and $1 \mathrm{~F}$ ). At the 12 and $24 \mathrm{~h}$ time points, glionitrin $\mathrm{A}$ caused $40.6 \pm 5.15$ and $60.9 \pm 4.55 \%$ apoptotic cell death compared with control showing $3.1 \pm 0.73 \%$ cell death, respectively. Together, these results demonstrate that glionitrin A inhibits the proliferation of DU145 cells by promoting cell cycle arrest and apoptosis.

The Effect of Glionitrin A on DNA DSBs DSBs are the most cytotoxic DNA lesions and also affect cell cycle progression and apoptosis. ${ }^{17,18)}$ Accordingly, we next explored whether glionitrin A causes DNA DSBs in DU145 cells. The nuclear DNA integrity was examined using a comet assay under neutral conditions. The level of DSBs in DU145 cells was analyzed after exposure to $3 \mu \mathrm{M}$ glionitrin A for $24 \mathrm{~h}$ (Fig. 2A). In the control group, the comet heads were concentrated with a high density of DNA, indicating genome integrity. In the glionitrin A treatment group, the comet heads were less

A

Control

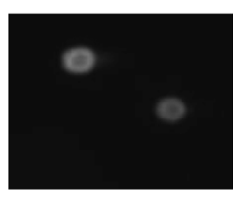

$3 \mu \mathrm{M}$ Glionitrin A

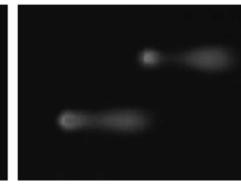

B



C



D

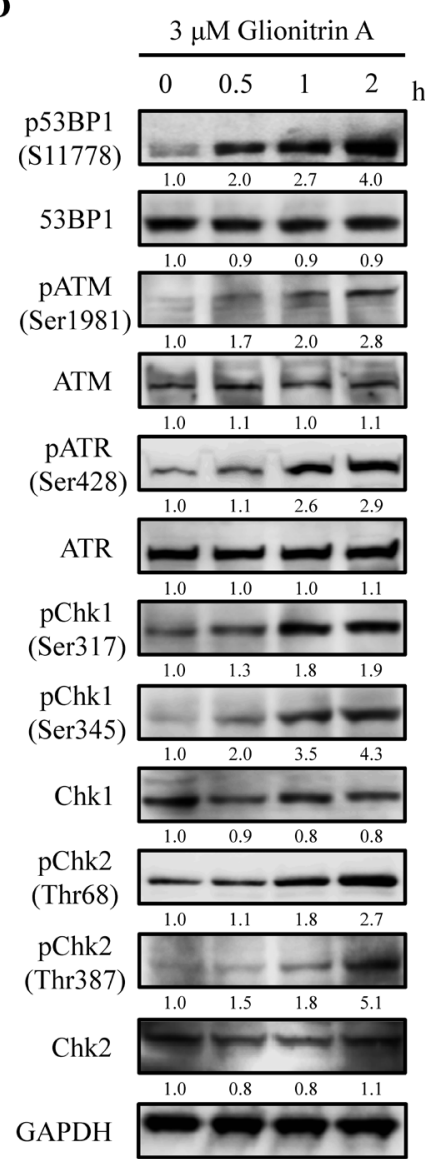

$\mathbf{E}$

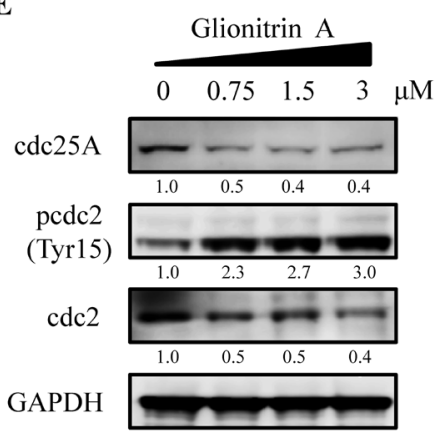

Fig. 2. Glionitrin A Elevates H2A.X Phosphorylation by Responding to DNA Damage Resulting from the Activation of ATM/ATR-Chk1/2 in a 53BP1-Dependent Manner

(A) Cells were treated with $3 \mu \mathrm{m}$ glionitrin A for $24 \mathrm{~h}$, and a comet assay was performed to confirm chromosomal DNA double-strand breaks. (B) The quantitative results of glionitrin A-induced tail moment. Each value represents the mean \pm S.E.M. of three independent experiments. (C) Cells were treated with the indicated concentrations of glionitrin A for $24 \mathrm{~h}$, and the protein levels of phospho-H2A.X were detected by Western blot analysis. (D) Cells were treated with $3 \mu \mathrm{M}$ glionitrin A for $24 \mathrm{~h}$, and the protein levels of phospho-53BP1, 53BP1, phospho-ATM, ATM, phospho-ATR, ATR, phospho-Chk1, Chk1, phospho-Chk2 and Chk2 were detected by western blot analysis using their respective antibodies. (E) Cells were treated with the indicated concentrations of glionitrin A for $24 \mathrm{~h}$, and the protein levels of cdc25A, phospho-cdc2 and cdc2 were detected by Western blot analysis using their respective antibodies. GAPDH was used as an internal control. A representative immunoblot of three independent experiments is shown. Density ratios over GAPDH were measured by densitometer. 
dense than those of the control and had broom-shaped tails. Figure $2 \mathrm{~B}$ shows the aspect of tail moment as a quantitative parameter for the analysis Fig. 2A. We next confirmed the phosphorylation status of H.X2A at Ser139, which is a sensitive marker of the early DNA damage response. As shown in Fig. 2C, exposure to glionitrin A for $24 \mathrm{~h}$ induced the phosphorylation of histone H2A.X in a dose-dependent manner.

The Effect of Glionitrin A on the Cell Cycle Checkpoint via 53BP1-ATM/ATR-Chk1/2 Signaling H2A.X is activated by the kinases ATM and ATR, which also phosphorylate various downstream substrates, such as Chk1/2. ${ }^{14}$ ) Phosphorylated Chk1/2 subsequently activates the kinase cdc25 and is responsible for $\mathrm{S}$ and/or G2/M phase checkpoint control. ${ }^{10,19)}$ Recent research has shown that 53BP1 performs multiple functions and importantly contributes to tumor suppression via pathways related to DNA repair, cell cycle control, and other factors. ${ }^{20)}$ Moreover, 53BP1 can also amplify ATM signals. ${ }^{21)}$ Based on these previous reports and the result shown in Fig. 2C, we performed western blotting to determine whether glionitrin A alters the levels of proteins related to 53BP1 signaling (Fig. 2D). Exposure of DU145 cells to $3 \mu \mathrm{M}$ glionitrin A resulted in a significant increase in the phosphorylation of 53BP1 in a time-dependent manner up to $2 \mathrm{~h}$. This response triggered the phosphorylation of ATM and ATR, which subsequently induced the phosphorylation of both Chk1 (Ser317 and Ser345) and Chk2 (Thr68 and Thr387), after $1 \mathrm{~h}$ of glionitrin A treatment. Additionally, glionitrin A treatment for $24 \mathrm{~h}$ caused cdc25A degradation and a strong phosphorylation of cdc2 at Tyr15 in a dose-dependent manner (Fig. 2E).

The results shown in Fig. 2 indicate that glionitrin A triggered a genotoxic stress response through 53BP1 activation, followed by the observed cell cycle arrest. To further substantiate these findings, DU145 cells were pretreated with $10 \mathrm{~mm}$ caffeine, an inhibitor of both ATM and ATR, for $2 \mathrm{~h}$ prior to glionitrin A treatment, and then cell cycle progression, the signaling pathways associated with checkpoint and cell proliferation were examined. As shown in Fig. 3A, caffeine completely reversed both the $\mathrm{S}$ and $\mathrm{G} 2 / \mathrm{M}$ phase accumulations induced by glionitrin A treatment to the control levels. Furthermore, the caffeine treatment markedly inhibited the glionitrin A-mediated phosphorylation of Chk1/2 (Fig. 3B). The caffeine treatment also restored the levels of cdc25A ex-


D
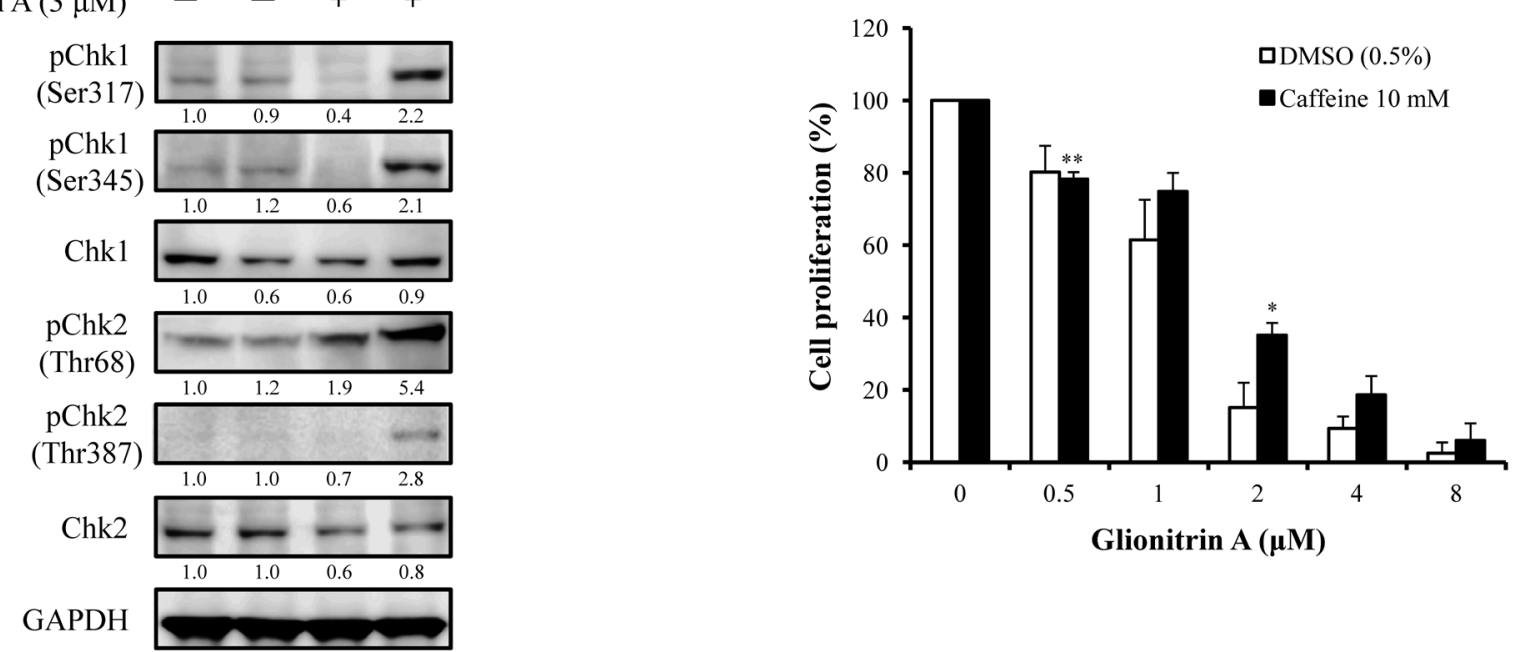

Fig. 3. Glionitrin A Causes Cell Cycle Arrest by the Activation of ATM-ATR Signaling

(A) Cells were pretreated with $10 \mathrm{~mm}$ caffeine for $2 \mathrm{~h}$ followed by exposure to $3 \mu \mathrm{m}$ glionitrin A for $24 \mathrm{~h}$, and FACS analysis/PI staining was then performed to assess cell cycle distribution. (B) Cells were pretreated with $10 \mathrm{~mm}$ caffeine for $2 \mathrm{~h}$ followed by exposure to $3 \mu \mathrm{M}$ glionitrin A for $2 \mathrm{~h}$, and the protein levels of phospho-Chk1, Chk1, phospho-Chk2 and Chk2 were detected by Western blot analysis using their respective antibodies. (C). Cells were pretreated with 10 mM caffeine for $2 \mathrm{~h}$ followed by exposure to $3 \mu \mathrm{M}$ glionitrin A for $24 \mathrm{~h}$, and the protein levels of cdc $25 \mathrm{~A}$, phospho-cdc 2 and cdc2 were detected by Western blot analysis using their respective antibodies. (D) Cells were pretreated with $10 \mathrm{~mm}$ caffeine for $2 \mathrm{~h}$ followed by exposure to the indicated concentrations of glionitrin A for $24 \mathrm{~h}$, and cell proliferation was examined by CCK- 8 assay. Each value represents the mean \pm S.E.M. of three independent experiments. $* p<0.05$ and $* * p<0.01$ compared with the control. GAPDH was used as an internal control. A representative immunoblot of three independent experiments is shown. Density ratios over GAPDH were measured by densitometer. 
pression and cdc2 phosphorylation to normal at the $24 \mathrm{~h}$ time point (Fig. 3C). To determine whether the cell cycle arrest resulting from the DNA damage response is associated with cell proliferation, we measured the cell proliferation after restoring the cell cycle to its original state via caffeine pretreatment. The glionitrin A-induced inhibition of cell proliferation was somewhat reduced by the caffeine pretreatment (Fig. 3D). These results suggest that glionitrin A activates ATM/ATRChk1/2 in a 53BP1-dependent manner, leading to cell cycle arrest involving cdc25A and $\operatorname{cdc} 2$, and that these events partially collaborate to inhibit cell proliferation.

The Effect of Glionitrin A on the Caspase-Dependent Apoptosis Pathway Because our results showed that glionitrin A induces apoptosis (Fig. 1E), we investigated whether caspase activation is involved in this induction. As shown in Fig. 4A, exposure to $3 \mu \mathrm{M}$ glionitrin A for $24 \mathrm{~h}$ induced the cleavage of PAR as well as that of caspase-3, -8 and -9 in a dose-dependent manner. Truncated Bid (tBid), which is cleaved at Asp60 by caspase- 8 during Fas signaling, and Bax were also elevated by glionitrin A. Next, we examined the effect of the pan-caspase inhibitor Z-VAD-fmk on cell proliferation to further confirm the role played by caspases in glionitrin A-induced apoptosis. DU145 cells were pretreated with $20 \mu \mathrm{M}$ Z-VAD-fmk for $2 \mathrm{~h}$ prior to glionitrin A treatment, and the activities of caspase-3, 8 and 9 were measured. Z-VADfmk completely inhibited glionitrin A-induced caspase activity to near basal levels (Fig. 4B). However, this inhibitory effect partially restored the decrease in cell proliferation caused by glionitrin A (Fig. 4C). These findings demonstrate that glionitrin A induces the activation of caspase-3, -8 and -9 , which contributes to apoptotic cell death.

The Effect of Glionitrin A on the Caspase-Independent Apoptosis Pathway Given that Z-VAD-fmk only partially rescues glionitrin A-induced apoptotic cell death, we next explored whether glionitrin A triggers the release of caspaseindependent apoptotic factors, such as AIF and EndoG, from the mitochondria into the nucleus. As shown in Fig. 5A, no changes in the nuclear translocation of AIF were detected after $24 \mathrm{~h}$ of glionitrin A treatment. However, there was a significant increase in the nuclear translocation of EndoG. Immunofluorescent imaging also showed that glionitrin A induced the release of EndoG from the mitochondria into the cytosol and nucleus (Fig. 5B). Z-VAD-fmk did not affect the glionitrin A-induced translocation of EndoG, confirming that this event is associated with the caspase-independent apoptosis pathway. Taken together, these results suggest that glionitrin A-induced apoptosis involves both the caspase-independent pathway and the caspase-dependent pathway.

The Effect of Glionitrin A on Tumor Growth in DU145Xenografted Nude Mice To study the antiproliferative activity of glionitrin A in vivo, we produced DU145 tumor xenografts in athymic nude mice. The mean tumor size in
A

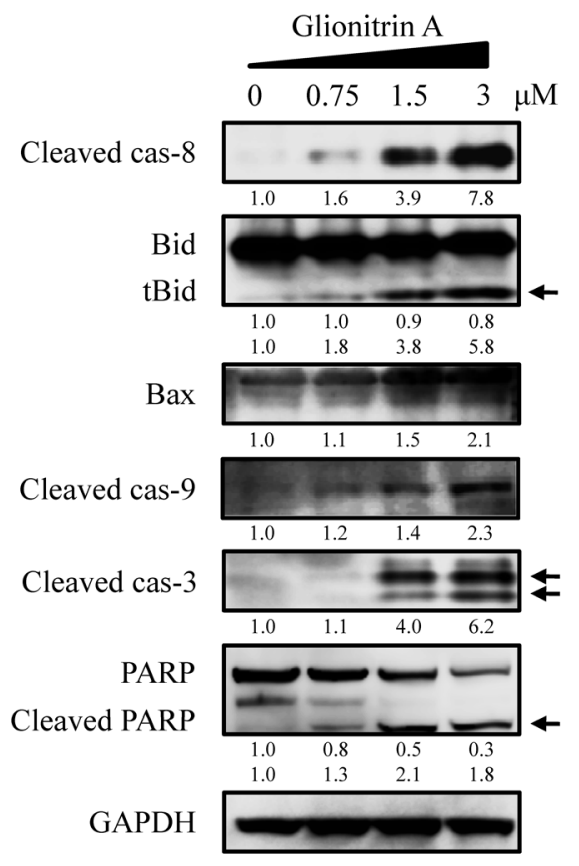

B



C

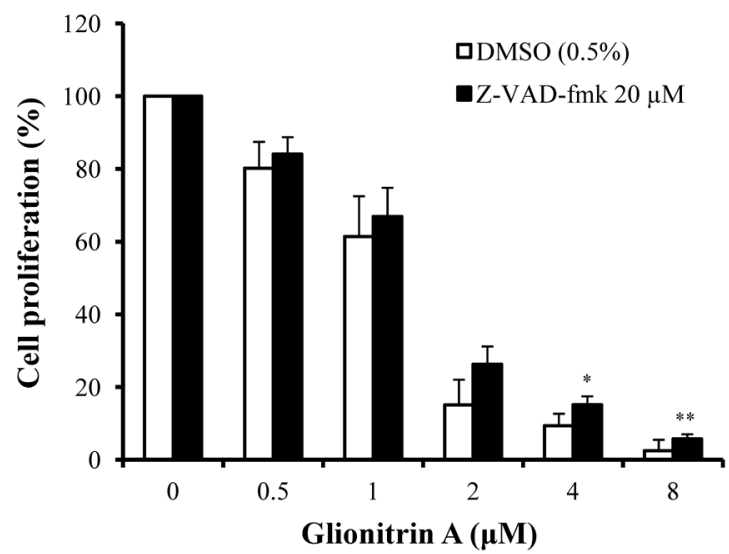

Fig. 4. Glionitrin A Induces Caspase-Dependent Apoptosis

(A) Cells were treated with the indicated concentrations of glionitrin A for $24 \mathrm{~h}$, and the protein levels of cleaved caspase- 8 , Bid, Bax, cleaved caspase- 9 , cleaved caspase-3 and PARP were detected by Western blot analysis using their respective antibodies. GAPDH was used as an internal control. A representative immunoblot of three independent experiments is shown. Density ratios over GAPDH were measured by densitometer. (B) Cells were pretreated with $20 \mu \mathrm{M} Z$-VAD-fmk for $2 \mathrm{~h}$ followed by exposure to $3 \mu \mathrm{m}$ glionitrin A for $24 \mathrm{~h}$, and the activity levels of caspase-3, -8 and -9 were then estimated. (C) Cells were pretreated with $20 \mu \mathrm{M} Z$-VAD-fmk for $2 \mathrm{~h}$ followed by exposure to the indicated concentrations of glionitrin A for $24 \mathrm{~h}$, and cell proliferation was then examined by CCK- 8 assay. Each value represents the mean \pm S.E.M. of three independent experiments. ${ }^{*} p<0.05$ and $* * p<0.01$ compared with the control. 
A

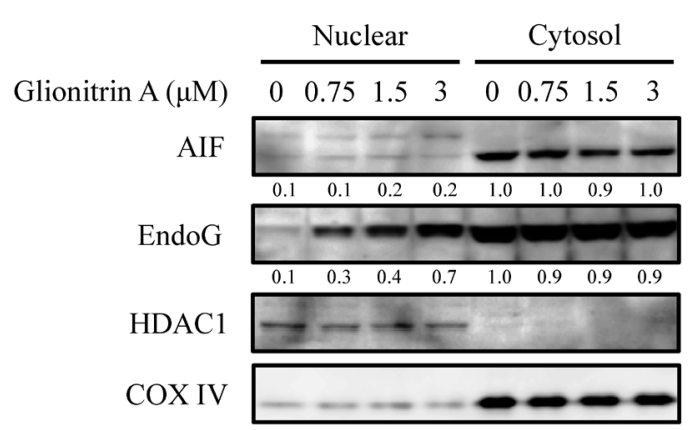

B



Fig. 5. Glionitrin A Induces Caspase-Independent Apoptosis

(A) Cytosolic and nuclear extracts were prepared from cells treated with the indicated concentrations of glionitrin A for $24 \mathrm{~h}$, and the protein levels of AIF and EndoG were detected by Western blot analysis using their respective antibodies. HDAC1 and COX IV were used as nuclear and cytosolic controls, respectively. A representative immunoblot of three independent experiments is shown. Density ratios over HDAC1 and COX IV were measured by densitometer. (B) Cells were pretreated with $20 \mu \mathrm{M}$ Z-VAD-fmk for $2 \mathrm{~h}$ followed by exposure to $3 \mu \mathrm{M}$ glionitrin A for $24 \mathrm{~h}$, and EndoG localization was then determined by confocal microscopy.

glionitrin A-treated mice was significantly smaller than that in the control group (Figs. 6A, B). At $27 \mathrm{~d}$ after the beginning of treatment, the average tumor volume in mice treated with $5 \mathrm{mg} / \mathrm{kg}$ glionitrin A was $61.8 \pm 3.69 \%$ of that in the mice in the control group, which is similar to the result obtained for mice treated with cisplatin $(64.9 \pm 7.43 \%$ of control). Additionally, the average tumor volume in mice treated with $10 \mathrm{mg} /$ $\mathrm{kg}$ glionitrin A was further reduced, to only $28.7 \%$ of that of the mice in the control group. The animals did not exhibit any considerable changes in body weight during the experimental period (Fig. 6C). These data show that glionitrin A markedly suppresses tumor growth in vivo and is more effective than cisplatin in this context.

\section{DISCUSSION}

A wide range of naturally occurring molecules have served as candidates of biological and chemical anticancer drugs in modern drug development schemes. ${ }^{22,23)}$ However, as the rate of known compound re-isolation from terrestrial plants and microorganisms has been increasing for more than a decade, ${ }^{24)}$ there is a need to develop more efficient methods for the discovery of new natural products that are more effective and less harmful than the existing therapies. Glionitrin A is a novel diketopiperazine disulfide that is produced during the competitive interaction between the bacteria Sphingomonas and the fungus Aspergillus fumigatus; this competitive interaction represents one of the more efficient methods for the production of new natural products. ${ }^{1)}$ The structure of glionitrin A is similar to that of gliotoxin, a compound isolated from Aspergillus that has been shown to cause DNA breakage, apoptosis and cell cycle arrest. ${ }^{25,26)}$ Therefore, we hypothesized that glionitrin A has a biological effect that is similar to that of gliotoxin and verified its anticancer potential using various in vitro experiments as well as an in vivo mouse model. In a preliminary study, we found that glionitrin A notably inhibited cell proliferation in various human cancer cell lines, especially human prostate cancer cells. Therefore, we used DU145 cells to further study the mechanisms underlying the antitumor effect of glionitrin A.

Following DNA damage, the autophosphorylation of 53BP1, a sensor of DNA DSBs that acts upstream of ATM, is responsible for the activation of ATM and ATR, which then phosphorylate downstream substrates, such as Chk1/2 and H2A.X. ${ }^{27,28)}$ The activation of Chk1/2 triggers the phosphorylation of the cdc25 phosphatase, which results in its binding to 14-3-3 and subsequent ubiquitin-mediated degradation, followed by the induction of cell cycle arrest. ${ }^{29)}$ During the $\mathrm{S}$ and $\mathrm{G} 2 / \mathrm{M}$ phases of the cell cycle, the cdc2-cyclin B complex is kept in its inactive form through the phosphorylation of cdc2 at Tyr15 by the Wee1/Mik1/Myt1 tyrosine kinases. ${ }^{30,31)}$ However, during the G2/M transition, cdc2 is rapidly activated by dephosphorylation at Tyr15 by the cdc25 phosphatase. ${ }^{32)}$ Several studies have reported that increased cdc2 phosphorylation at Tyr15 is closely linked to cell cycle arrest, especially during $\mathrm{S}$ and/or G2/M phase. ${ }^{19,33)}$ In this study, we investigated whether 
A



C



B

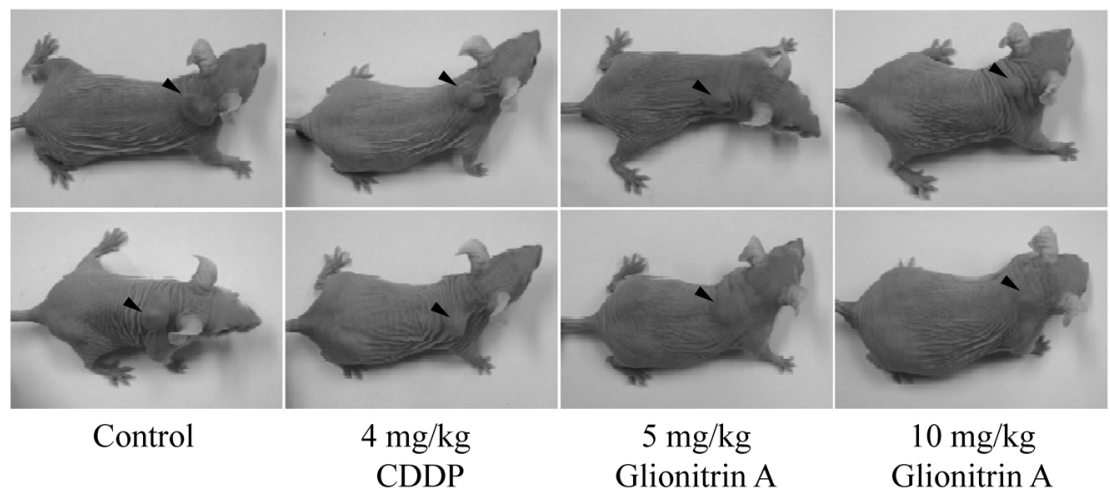

Fig. 6. Glionitrin A Inhibits Tumor Growth in Nude Mice Xenografted with DU145 Cells

Athymic nude mice were implanted with $1 \times 10^{7}$ DU145 cells on the right flank. When tumors had developed to approximately $80 \mathrm{~mm}^{3}$ in size, animals were fed glionitrin A $(5 \mathrm{mg} / \mathrm{kg}$ or $10 \mathrm{mg} / \mathrm{kg}$, p.o., twice a week for two weeks) or injected with cisplatin (CDDP; $4 \mathrm{mg} / \mathrm{kg}$, i.v., once a week for three weeks). Tumor sizes (A) and (B) and body weights (C) were measured twice a week. Points, means of seven values; bars, S.E.M.; * $p<0.05 ; * * p<0.01 ; * * *<<0.001$ compared with the control.

glionitrin A activates the DNA damage response as a possible mechanism for the cytotoxicity and cell cycle arrest in DU145 cells. We found that glionitrin A induced DNA DSBs, as evidenced by comet tail formation and increased H2A.X phosphorylation. In addition, we showed that glionitrin A induced ATM/ATR activation in a 53BP1-dependent manner, causing Chk1/2 phosphorylation, cdc $25 \mathrm{~A}$ degradation and the inhibition of cdc2 dephosphorylation. These events ultimately triggered $\mathrm{S}$ and $\mathrm{G} 2 / \mathrm{M}$ phase arrest. Conversely, the inhibition of ATM/ATR activation by caffeine rescued the downstream substrates and cell cycle progression to a normal state and partially restored the decrease in cell proliferation caused by glionitrin A. Thus, glionitrin A-induced DNA damage activates the cell cycle checkpoint through a mechanism that involves the 53BP1-ATM/ATR-Chk1/2-cdc25A-cdc2 cascade and partially contributes to the inhibition of cell proliferation.

The DNA damage response often induces extrinsic death receptor-dependent apoptosis and/or intrinsic mitochondrial apoptosis through both p53-dependent and p53-independent pathways, depending on the DNA damage agent and cell type. ${ }^{1434)}$ During Fas signaling, Bid is cleaved at Asp60 by activated caspase- 8 , leading to the translocation of the carboxyl terminal p15 fragment (tBid) from the cytosol to the mitochondrial outer membrane. ${ }^{35,36)}$ The interaction between tBid and Bax is associated with the release of cytochrome c from the mitochondria, leading to the activation of caspase-3 and $-9 .{ }^{37)}$ Consistent with previous observations, our data showed that glionitrin A activated caspase-8, leading to increased levels of tBid and Bax and subsequent PARP cleavage through the activation of caspase-3 and -9 in DU145 cells. Surprisingly, the glionitrin A-induced apoptotic pathway in DU145 cells seemed to be involved ATM-p53 signaling, even though DU145 is known as a p53 mutant cell line. Further study is necessary to investigate this phenomenon. To further confirm the role played by caspases in glionitrin A-induced apoptosis, we investigated whether the inhibition of caspase activity restores the cell proliferation phenotype. Z-VAD-fmk partially relieved glionitrin A-induced cell death, suggesting that the apoptosis induced by glionitrin A is partially mediated by caspases. Mammalian mitochondria harbor enzymes with strong nuclease activity, which is evident when the membranes become dysfunctional or are disrupted by detergents. ${ }^{38)}$ EndoG, a mitochondrion-specific nuclease, is translocated to the nucleus during apoptosis. Once inside the nucleus, EndoG cleaves the chromatin into nucleosomal fragments in a caspase-independent manner. ${ }^{39)}$ Based on previous reports, we also investigated whether glionitrin A causes the release of caspase-independent apoptotic factors. Interestingly, we observed a significant increase in the nuclear translocation of EndoG but not AIF. These results show that glionitrin Ainduced apoptotic cell death is associated with both caspasedependent and caspase-independent pathways.

Finally, to verify the in vivo relevance to the in vitro biological activity of glionitrin A, we investigated whether glionitrin A has antitumor activity in vivo using DU145 cell xenografts in athymic nude mice as a representative in vivo model. We found that treatment with glionitrin A significantly suppressed tumor growth with no change in animal body weight. 
In summary, our results provide the first evidence that glionitrin A, a new diketopiperazine disulfide, induces the DNA damage response in DU145 cells, promoting cell cycle arrest and apoptosis. Thus, glionitrin A could be a useful pharmacological tool with which to enhance our understanding of basic cellular functions and could also contribute to the development of a new class of therapeutic or chemopreventive drugs for human prostate cancer.

Acknowledgments This study was supported by the KIST Institutional Program (2Z03840) and Bio-Synergy Research Project (NRF-2013M3A9C4078142) of the Ministry of Science, ICT and Future Planning through the National Research Foundation.

\section{REFERENCES}

1) Park HB, Kwon HC, Lee CH, Yang HO. Glionitrin A, an antibioticantitumor metabolite derived from competitive interaction between abandoned mine microbes. J. Nat. Prod., 72, 248-252 (2009).

2) Waring P, Beaver J. Gliotoxin and related epipolythiodioxopiperazines. Gen. Pharmacol., 27, 1311-1316 (1996).

3) Gardiner DM, Waring P, Howlett BJ. The epipolythiodioxopiperazine (ETP) class of fungal toxins: distribution, mode of action, functions and biosynthesis. Microbiology, 151, 1021-1032 (2005).

4) Fulda S, Debatin KM. Apoptosis signaling in tumor therapy. Ann. N. Y. Acad. Sci., 1028, 150-156 (2004).

5) Norbury CJ, Zhivotovsky B. DNA damage-induced apoptosis. Oncogene, 23, 2797-2808 (2004).

6) Ramaiah MJ, Pushpavalli S, Krishna GR, Sarma P, Mukhopadhyay D, Kamal A, Bhadra U, Bhadra MP. Chalcone-imidazolone conjugates induce apoptosis through DNA damage pathway by affecting telomeres. Cancer Cell Int., 11, 11 (2011).

7) Loeb KR, Loeb LA. Significance of multiple mutations in cancer. Carcinogenesis, 21, 379-385 (2000).

8) Abraham RT. Cell cycle checkpoint signaling through the ATM and ATR kinases. Genes Dev., 15, 2177-2196 (2001).

9) Kastan MB, Bartek J. Cell-cycle checkpoints and cancer. Nature, 432, 316-323 (2004).

10) Liu Q, Guntuku S, Cui XS, Matsuoka S, Cortez D, Tamai K, Luo G, Carattini-Rivera S, DeMayo F, Bradley A, Donehower LA, Elledge SJ. Chk1 is an essential kinase that is regulated by Atr and required for the G(2)/M DNA damage checkpoint. Genes Dev., 14, 1448-1459 (2000).

11) Rogakou EP, Boon C, Redon C, Bonner WM. Megabase chromatin domains involved in DNA double-strand breaks in vivo. J. Cell Biol., 146, 905-916 (1999).

12) Kastan MB, Lim D-S, Kim S-T, Xu B, Maser RS, Lin J, Petrini JHJ. ATM phosphorylates p95/nbs1 in an S-phase checkpoint pathway. Nature, 404, 613-617 (2000).

13) Lane DP. Cancer. p53, guardian of the genome. Nature, 358, 15-16 (1992).

14) Roos WP, Kaina B. DNA damage-induced cell death by apoptosis. Trends Mol. Med., 12, 440-450 (2006).

15) Urist M, Tanaka T, Poyurovsky MV, Prives C. p73 induction after DNA damage is regulated by checkpoint kinases Chk1 and Chk2. Genes Dev., 18, 3041-3054 (2004).

16) Melino G, Bernassola F, Ranalli M, Yee K, Zong WX, Corazzari M, Knight RA, Green DR, Thompson C, Vousden KH. p73 Induces apoptosis via PUMA transactivation and Bax mitochondrial translocation. J. Biol. Chem., 279, 8076-8083 (2003).

17) Jackson SP. Sensing and repairing DNA double-strand breaks. Carcinogenesis, 23, 687-696 (2002).

18) Lavin MF, Birrell G, Chen P, Kozlov S, Scott S, Gueven N. ATM signaling and genomic stability in response to DNA damage. Mutat. Res., 569, 123-132 (2005).

19) Agarwal C, Tyagi A, Agarwal R. Gallic acid causes inactivating phosphorylation of cdc25A/cdc25C-cdc2 via ATM-Chk2 activation, leading to cell cycle arrest, and induces apoptosis in human prostate carcinoma DU145 cells. Mol. Cancer Ther., 5, 3294-3302 (2006).

20) Kass EM, Moynahan ME, Jasin M. Loss of 53BP1 is a gain for BRCA1 mutant cells. Cancer Cell, 17, 423-425 (2010).

21) Lee JH, Goodarzi AA, Jeggo PA, Paull TT. 53BP1 promotes ATM activity through direct interactions with the MRN complex. $E M B O$ J., 29, 574-585 (2010).

22) Mann J. Natural products in cancer chemotherapy: past, present and future. Nat. Rev. Cancer, 2, 143-148 (2002).

23) Koehn FE, Carter GT. The evolving role of natural products in drug discovery. Nat. Rev. Drug Discov., 4, 206-220 (2005).

24) Corley DG, Miller-Wideman M, Durley RC. Isolation and structure of harzianum A: a new trichothecene from Trichoderma harzianum. J. Nat. Prod., 57, 422-425 (1994).

25) Braithwaite AW, Eichner RD, Waring P, Müllbacher A. The immunomodulating agent gliotoxin causes genomic DNA fragmentation. Mol. Immunol., 24, 47-55 (1987).

26) Waring P, Mamchak A, Khan T, Sjaarda A, Sutton P. DNA synthesis precedes gliotoxin-induced apoptosis. Cell Death Differ., 2 , 201-210 (1995).

27) Bakkenist CJ, Kastan MB. DNA damage activates ATM through intermolecular autophosphorylation and dimer dissociation. Nature, 421, 499-506 (2003).

28) Mochan TA, Venere M, DiTullio RA Jr, Halazonetis TD. 53BP1 and NFBD1/MDC1-Nbs1 function in parallel interacting pathways activating ataxia-telangiectasia mutated (ATM) in response to DNA damage. Cancer Res., 63, 8586-8591 (2003).

29) Chen MS, Ryan CE, Piwnica-Worms H. Chk1 kinase negatively regulates mitotic function of Cdc25A phosphatase through 14-3-3 binding. Mol. Cell. Biol., 23, 7488-7497 (2003).

30) Parker LL, Piwnica-Worms H. Inactivation of the p34cdc2-cyclin B complex by the human WEE1 tyrosine kinase. Science, 257, 1955-1957 (1992).

31) Mueller PR, Coleman TR, Dunphy WG. Cell cycle regulation of a Xenopus Weel-like kinase. Mol. Biol. Cell, 6, 119-134 (1995).

32) Kumagai A, Dunphy WG. The cdc25 protein controls tyrosine dephosphorylation of the cdc2 protein in a cell-free system. Cell, $\mathbf{6 4}$, 903-914 (1991).

33) Jia XZ, Yang SY, Zhou J, Li SY, Ni JH, An GS, Jia HT. Inhibition of CHK1 kinase by Gö6976 converts 8-chloro-adenosine-induced G2/M arrest into S arrest in human myelocytic leukemia K562 cells. Biochem. Pharmacol., 77, 770-780 (2009).

34) Wichmann A, Jaklevic B, Su TT. Ionizing radiation induces caspase-dependent but Chk2- and p53-independent cell death in Drosophila melanogaster. Proc. Natl. Acad. Sci. U.S.A., 103, 99529957 (2006).

35) Li H, Zhu H, Xu CJ, Yuan J. Cleavage of BID by caspase 8 mediates the mitochondrial damage in the Fas pathway of apoptosis. Cell, 94, 491-501 (1998).

36) Luo X, Budihardjo I, Zou H, Slaughter C, Wang X. Bid, a Bcl2 interacting protein, mediates cytochrome $c$ release from mitochondria in response to activation of cell surface death receptors. Cell, 94, 481-490 (1998).

37) Billen LP, Kokoski CL, Lovell JF, Leber B, Andrews DW. Bcl-XL inhibits membrane permeabilization by competing with Bax. PLoS Biol., 6, e147 (2008).

38) Ohsato T, Ishihara $N$, Muta $T$, Umeda S, Ikeda S, Mihara K, Hamasaki N, Kang D. Mammalian mitochondrial endonuclease G. Digestion of R-loops and localization in intermembrane space. Eur. J. Biochem., 269, 5765-5770 (2002).

39) Li LY, Luo $X$, Wang $X$. Endonuclease $G$ is an apoptotic DNase when released from mitochondria. Nature, 412, 95-99 (2001). 